\title{
Origin of Gauge Theories in Electrodynamics
}

\author{
Jay Solanki
}

\begin{abstract}
The potential formulation has significant advantages over field formulation in solving complicated problems in electromagnetic field theory. One essential part of electromagnetic field theory's potential formulation is gauge invariance and gauge theories because it provides an extra degree of freedom. By using this extra degree of freedom, we can solve complicated electromagnetic problems quickly. Thus, it is necessary to include a systematic explanation of gauge theories in teaching electromagnetic theory. However, textbooks usually formulate gauge theories by using Maxwell's electromagnetism equations by using vector calculus identities. However, this method of formulation of gauge theories does not give a clear idea about gauge theories' origin and gauge invariance in electromagnetism. Here the author formulates gauge theories from wave equations of the electric and magnetic fields instead of directly using Maxwell's equations. This method generalizes all gauge theories like Lorenz gauge theory, Coulomb gauge theory, Etc. Gauge theory, because of the way the author derives it, gives a distinct idea about the mathematical origin of the gauge theories and gauge invariance in electromagnetic field theory. Thus, the author reviews the origin of gauge theories in electromagnetic field theory and develops a distinct and effective method to introduce gauge theory in the teaching of electromagnetic field theory that can provide a better understanding of the topic to undergraduate students.
\end{abstract}

. Index Terms-Maxwell's equations, electromagnetic field, Helmholtz theorem, gauge theory

\section{INTRODUCTION}

$\mathbf{I}$ $\mathrm{T}$ It is well known that Maxwell's four electromagnetism equations describe the electromagnetic field produced by charges and currents. By solving these four Maxwell's equations, we can find electromagnetic fields produced by given charges and currents. There are two popular methods of solving these equations; the first is to use the gauge theories like Lorenz gauge theory, Coulomb gauge theory, Etc.[1, pp. 237248], and the second one is to use the Helmholtz theorem.[2, p. 3]. Some papers appeared in the TRANSACTIONS concerned with a simplified representation of electromagnetic field theory [3] and geometrical approach to electromagnetic field theory [4]. Modern approach in the teaching of electromagnetic field theory by potentials[5] and differential forms [6] also presented in TRANSACTIONS. Those papers formulated electromagnetic field theory by direct use of gauge invariance and gauge theories. However, It is essential to formulate electromagnetic field theory such that the origin of gauge theories reflects naturally in the mathematical formulation of the theory in the teaching of the subject. Thus here, the author reformulates electromagnetic field theory such that the origin of gauge theory naturally justifies by the mathematical use of vector calculus properties. Thus, it can be valuable and effective in introducing the topic in the teaching of the subject.

The author is with Bose.X Scientific Research Group, Sardar Vallabhbhai National Institute of Technology,Surat,Gujarat,395007,India. e-mail: (jay565109@gmail.com).
Now, it is necessary to use generalized wave equations of the electric and magnetic field to formulate gauge theory instead of directly using Maxwell's equations to clearly understand the gauge theory's mathematical origin in electromagnetic field theory. That is why the author first derives generalized wave equations of the electric and magnetic field by solving Maxwell's electromagnetism equations using the Helmholtz theorem.

The purpose of re-deriving wave equations of the electric and magnetic field from Maxwell's equations is to present a precise and complete formulation of the subject. To fulfill this purpose, in section II, the author derives the wave equation of the electric and magnetic field from Maxwell's equations by using the Helmholtz theorem. In section III, the author formulates generalized potential equations from field equations(wave equations of the electric and magnetic field). Generalized potential equations that the author derives in section III represents generalized gauge theory. In section IV, the author formulates the Lorenz gauge theory and Coulomb gauge theory from Generalized potential equations. However, the author shows only two gauge theories, but generalized potential equations represent many gauge theories.

\section{Derivation of Wave Equation from Maxwell's EQUATIONS USING HelMHOLTZ THEOREM}

Maxwell's four equations of electromagnetism in SI units are[7. p. 337]

$$
\begin{gathered}
\nabla \cdot \mathbf{E}=\frac{\rho}{\epsilon_{0}} \\
\nabla \times \mathbf{E}=-\frac{\partial \mathbf{B}}{\partial t} \\
\nabla \cdot \mathbf{B}=0 \\
\nabla \times \mathbf{B}=\mu_{0} \mathbf{J}+\mu_{0} \epsilon_{0} \frac{\partial \mathbf{E}}{\partial t}
\end{gathered}
$$

Where electric and magnetic field respectively $\mathbf{E}$ and $\mathbf{B}$ are produced by charge and current densities respectively $\rho$ and J. $\epsilon_{0}$ and $\mu_{0}$ are permittivity and permeability constants of the vacuum which satisfy $\epsilon_{0} \mu_{0}=\frac{1}{c^{2}}$.

According to Helmholtz theorem any vector field $\mathbf{F}(\mathbf{r}, t)$ that goes to zero faster than $\frac{1}{r}$ as $\mathrm{r} \rightarrow \infty$ can be expressed as 7 . pp. 582-584]

$$
\mathbf{F}(\mathbf{r}, t)=-\nabla \int \frac{\nabla^{\prime} \cdot \mathbf{F}\left(\mathbf{r}^{\prime}, t\right)}{4 \pi R} d^{3} r^{\prime}+\nabla \times \int \frac{\nabla^{\prime} \times \mathbf{F}\left(\mathbf{r}^{\prime}, t\right)}{4 \pi R} d^{3} r^{\prime}
$$

where the integral are extended over all space, $R=\left|\mathbf{r}-\mathbf{r}^{\prime}\right|$ with $\mathbf{r}$ being the field point and $\mathbf{r}^{\prime}$ the source point.

By using the Helmholtz theorem, the electric field can be written as 


$$
\mathbf{E}(\mathbf{r}, t)=-\nabla \int \frac{\nabla^{\prime} \cdot \mathbf{E}\left(\mathbf{r}^{\prime}, t\right)}{4 \pi R} d^{3} r^{\prime}+\nabla \times \int \frac{\nabla^{\prime} \times \mathbf{E}\left(\mathbf{r}^{\prime}, t\right)}{4 \pi R} d^{3} r^{\prime}
$$

Now using Maxwell's equations (1) and (2), the above equation can be written as

$\mathbf{E}(\mathbf{r}, t)=-\nabla \int \frac{1}{4 \pi \epsilon_{0}} \frac{\rho\left(\mathbf{r}^{\prime}, t\right)}{R} d^{3} r^{\prime}+\nabla \times \int \frac{1}{4 \pi R}\left(-\frac{\partial \mathbf{B}\left(\mathbf{r}^{\prime}, t\right)}{\partial t}\right)$

Introduce the operator $\nabla \times$ into the second integral in the above equation, use Maxwell's equation (4), and perform integration by parts to obtain the expression

$$
\begin{aligned}
\mathbf{E}(\mathbf{r}, t) & =-\nabla \int \frac{1}{4 \pi \epsilon_{0}} \frac{\rho\left(\mathbf{r}^{\prime}, t\right)}{R} d^{3} r^{\prime} \\
& -\frac{\partial}{\partial t} \int \frac{1}{4 \pi R}\left(\mu_{0} \mathbf{J}\left(\mathbf{r}^{\prime}, t\right)+\mu_{0} \epsilon_{0} \frac{\partial \mathbf{E}\left(\mathbf{r}^{\prime}, t\right)}{\partial t}\right) d^{3} r^{\prime} \\
\mathbf{E}(\mathbf{r} . t)= & -\nabla \int \frac{1}{4 \pi \epsilon_{0}} \frac{\rho\left(\mathbf{r}^{\prime}, t\right)}{R} d^{3} r^{\prime} \\
& -\frac{\mu_{0}}{4 \pi} \frac{\partial}{\partial t} \int \frac{\mathbf{J}\left(\mathbf{r}^{\prime}, t\right)}{4 \pi R} d^{3} r^{\prime}-\frac{1}{c^{2}} \frac{\partial^{2}}{\partial t^{2}} \int \frac{\mathbf{E}\left(\mathbf{r}^{\prime}, t\right)}{4 \pi R} d^{3} r^{\prime}
\end{aligned}
$$

By applying $\nabla^{2}$ operator to above equation, use $\nabla^{2}\left(\frac{1}{R}\right)=$ $-4 \pi \delta\left(\mathbf{r}-\mathbf{r}^{\prime}\right)$, where $\delta$ is the Dirac delta function, and integrate overall space then we obtain the familiar wave equation for electric field

$$
\nabla^{2} \mathbf{E}(\mathbf{r}, t)=\frac{\nabla \rho(\mathbf{r}, t)}{\epsilon_{0}}+\mu_{0} \frac{\partial \mathbf{J}(\mathbf{r}, t)}{\partial t}+\frac{1}{c^{2}} \frac{\partial^{2} \mathbf{E}(\mathbf{r}, t)}{\partial t^{2}}
$$

equation (10) can be simply written as

$$
\nabla^{2} \mathbf{E}-\frac{1}{c^{2}} \frac{\partial^{2} \mathbf{E}}{\partial t^{2}}=\frac{\nabla \rho}{\epsilon_{0}}+\mu_{0} \frac{\partial \mathbf{J}}{\partial t}
$$

Equation (11) is a wave equation for electric field with source charge and current densities.[1, p. 246]

Now by Helmholtz theorem, magnetic field can be written as

$$
\mathbf{B}(\mathbf{r}, t)=-\nabla \int \frac{\nabla^{\prime} \cdot \mathbf{B}\left(\mathbf{r}^{\prime}, t\right)}{4 \pi R} d^{3} r^{\prime}+\nabla \times \int \frac{\nabla^{\prime} \times \mathbf{B}\left(\mathbf{r}^{\prime}, t\right)}{4 \pi R} d^{3} r^{\prime}
$$

by using Maxwell's equation (3) and (4),

$$
\mathbf{B}(\mathbf{r}, t)=\nabla \times \int \frac{1}{4 \pi R}\left(\mu_{0} \mathbf{J}\left(\mathbf{r}^{\prime}, t\right)+\mu_{0} \epsilon_{0} \frac{\partial \mathbf{E}\left(\mathbf{r}^{\prime}, t\right)}{\partial t}\right) d^{3} r^{\prime}
$$

By applying $\nabla^{2}$ operator to above equation, use $\nabla^{2}\left(\frac{1}{R}\right)=$ $-4 \pi \delta\left(\mathbf{r}-\mathbf{r}^{\prime}\right)$, where $\delta$ is the Dirac delta function, and integrate over all space then we obtain the familiar wave equation for magnetic field

$$
\nabla^{2} \mathbf{B}(\mathbf{r}, t)=-\mu_{0}(\nabla \times \mathbf{J})-\frac{1}{c^{2}} \frac{\partial}{\partial t}\left(-\frac{\partial \mathbf{B}}{\partial t}\right)
$$

Above equation can be simply written as

$$
\nabla^{2} \mathbf{B}-\frac{1}{c^{2}} \frac{\partial^{2} \mathbf{B}}{\partial t^{2}}=-\mu_{0}(\nabla \times \mathbf{J})
$$

Equation (15) is wave equation for magnetic field with source.[1, p. 246]

\section{Formulation of Generalized Potential Equations FROM FIELD EQUATIONS}

In the previous section, equations (11) and (15) represent wave equations of the electric and magnetic fields. By solving equations (11) and (15), we can directly get the electromagnetic field's value at every point of space-time. However, the complexity in the distribution of charges and currents, equations (11) and (15) becomes very complex to solve. However, one prevalent method to solve this problem is to use potential. For this, first, we have to obtain a relation between fields and potentials. For this, we can use Maxwell's equations (1) to (4).

Because of divergence of the magnetic field is zero in equation (3), we can write magnetic field in terms of vector potential $\mathbf{A}$ as

$$
\mathbf{B}=\nabla \times \mathbf{A}
$$

From equation (2) and (16) we can write

$$
\begin{gathered}
\nabla \times \mathbf{E}=-\frac{\partial}{\partial t}(\nabla \times \mathbf{A}) \\
\nabla \times\left(\mathbf{E}+\frac{\partial \mathbf{A}}{\partial t}\right)=0
\end{gathered}
$$

The solution of above equation is

$$
\mathbf{E}=-\nabla \phi-\frac{\partial \mathbf{A}}{\partial t}
$$

Where it is well known that $\phi$ is scalar potential.

Equation (16) and (19) represent the relation between fields and potentials.[1, p. 239]

So now we have the electric and magnetic field in terms of scalar and vector potential. We want to formulate equations that can give scalar and vector potential values at every point in space-time. We can formulate potential equations by applying equations (16) to (15) and (19) to (11).

From equation (16), wave equation of magnetic field - (15) can be written as

$$
\begin{gathered}
\nabla^{2}(\nabla \times \mathbf{A})-\frac{1}{c^{2}} \nabla \times\left(\frac{\partial^{2} \mathbf{A}}{\partial t^{2}}\right)=-\mu_{0}(\nabla \times \mathbf{J}) \\
\nabla \times\left(\nabla^{2} \mathbf{A}-\frac{1}{c^{2}} \frac{\partial^{2} \mathbf{A}}{\partial t^{2}}+\mu_{0} \mathbf{J}\right)=0
\end{gathered}
$$

The solution of above equation is

$$
\frac{1}{c^{2}} \frac{\partial^{2} \mathbf{A}}{\partial t^{2}}-\nabla^{2} \mathbf{A}=\mu_{0} \mathbf{J}+\nabla f
$$

In above equation $f(\mathbf{r}, t)$ is arbitrary scalar function.Above equation is generalized wave equation for vector potential.

Now by applying equation (19) into wave equation of electric field (11), we get,

$$
\nabla^{2}\left(-\nabla \phi-\frac{\partial \mathbf{A}}{\partial t}\right)-\frac{1}{c^{2}} \frac{\partial^{2}}{\partial t^{2}}\left(-\nabla \phi-\frac{\partial \mathbf{A}}{\partial t}\right)=\frac{\nabla \rho}{\epsilon_{0}}+\mu_{0} \frac{\partial \mathbf{J}}{\partial t}
$$




$$
\begin{aligned}
&-\nabla\left(\nabla^{2} \phi\right)+ \nabla\left(\frac{1}{c^{2}} \frac{\partial^{2} \phi}{\partial t^{2}}\right)-\nabla\left(\frac{\rho}{\epsilon_{0}}\right) \\
&+\frac{\partial}{\partial t}\left(\frac{1}{c^{2}} \frac{\partial^{2} \mathbf{A}}{\partial t^{2}}-\nabla \times \mathbf{A}-\mu_{0} \mathbf{J}\right)=0 \\
& \nabla\left(\frac{1}{c^{2}} \frac{\partial^{2} \phi}{\partial t^{2}}-\nabla^{2} \phi-\frac{\rho}{\epsilon_{0}}+\frac{\partial f}{\partial t}\right)=0
\end{aligned}
$$

The solution of above equation is,

$$
\frac{1}{c^{2}} \frac{\partial^{2} \phi}{\partial t^{2}}-\nabla^{2} \phi=\frac{\rho}{\epsilon_{0}}-\frac{\partial f}{\partial t}
$$

The above equation is a generalized wave equation of scalar potential.

Generalized wave equations for scalar and vector potential, as the author have derived are,

$$
\begin{gathered}
\frac{1}{c^{2}} \frac{\partial^{2} \mathbf{A}}{\partial t^{2}}-\nabla^{2} \mathbf{A}=\mu_{0} \mathbf{J}+\nabla f \\
\frac{1}{c^{2}} \frac{\partial^{2} \phi}{\partial t^{2}}-\nabla^{2} \phi=\frac{\rho}{\epsilon_{0}}-\frac{\partial f}{\partial t}
\end{gathered}
$$

In the above equations of scalar and vector potential, the scalar function " $f$ " is arbitrary. By choosing different values of "f" we can get many different gauges like Lorenz gauge, Coulomb gauge, Etc.

In the next section, the author, formulates Lorenz and Coulomb gauge theories by choosing particular values of scalar function "f".

\section{Formulation of Lorenz and Coulomb Gauge} Theories from Generalized WaVe Equations of Scalar ANd Vector Potential

\section{A. Lorenz Gauge Theory from Generalized Wave Equations of Potential}

Since scalar function " $\mathrm{f}$ " in equations (27) and (28) is arbitrary, we can choose any value of it. In order to formulate Lorenz Gauge theory, choose $\mathrm{f}$ to be 0 , so that equation (27) and (28) becomes,

$$
\begin{gathered}
\frac{1}{c^{2}} \frac{\partial^{2} \mathbf{A}}{\partial t^{2}}-\nabla^{2} \mathbf{A}=\mu_{0} \mathbf{J} \\
\frac{1}{c^{2}} \frac{\partial^{2} \phi}{\partial t^{2}}-\nabla^{2} \phi=\frac{\rho}{\epsilon_{0}}
\end{gathered}
$$

It is well known that equation (29) and (30) are Lorenz equations of potentials [1, p. 240-242]

By applying $\frac{\partial}{\partial t}$ operator to the equation (30), we get,

$$
\frac{1}{c^{4}} \frac{\partial^{3} \phi}{\partial t^{3}}-\frac{1}{c^{2}} \nabla^{2}\left(\frac{\partial \phi}{\partial t}\right)=\frac{1}{c^{2} \epsilon_{0}} \frac{\partial \rho}{\partial t}
$$

By applying $\nabla$. operator to the equation (29) we get,

$$
\frac{1}{c^{2}} \frac{\partial^{2}(\nabla \cdot \mathbf{A})}{\partial t^{2}}-\nabla^{2}(\nabla \cdot \mathbf{A})=\mu_{0} \nabla \cdot \mathbf{J}
$$

By adding equations (31) and (32),

$$
\begin{gathered}
\frac{1}{c^{2}} \frac{\partial^{2}}{\partial t^{2}}\left(\frac{1}{c^{2}} \frac{\partial \phi}{\partial t}+\nabla \cdot \mathbf{A}\right)-\nabla^{2}\left(\frac{1}{c^{2}} \frac{\partial \phi}{\partial t}+\nabla \cdot \mathbf{A}\right)=\mu_{0}\left(\nabla \cdot \mathbf{J}+\frac{\partial \rho}{\partial t}\right) \\
\left(\frac{1}{c^{2}} \frac{\partial^{2}}{\partial t^{2}}-\nabla^{2}\right)\left(\frac{1}{c^{2}} \frac{\partial \phi}{\partial t}+\nabla \cdot \mathbf{A}\right)=0
\end{gathered}
$$

The solution of above equation is,

$$
\frac{1}{c^{2}} \frac{\partial \phi}{\partial t}+\nabla \cdot \mathbf{A}=0
$$

It is well known that the above equation is of Lorenz gauge[1, p. 240-242]

\section{B. Coulomb Gauge Theory from Generalized Wave Equations of Potential}

In order to formulate Coulomb gauge theory let,

$$
f=-\frac{1}{c^{2}} \frac{\partial \phi}{\partial t}
$$

By putting above value of $f$ in equation (28),

$$
\nabla^{2} \phi=-\frac{\rho}{\epsilon_{0}}
$$

Above equation is well known as Coulomb equation of scalar potential.[1, p. 240-242]

By applying $\nabla$ operator to " $\mathrm{f}$ " and using equation (19)

$$
\begin{gathered}
\nabla f=-\frac{1}{c^{2}} \frac{\partial}{\partial t}\left(-\mathbf{E}-\frac{\partial \mathbf{A}}{\partial t}\right) \\
\nabla f=\frac{1}{c^{2}} \frac{\partial \mathbf{E}}{\partial t}+\frac{1}{c^{2}} \frac{\partial^{2} \mathbf{A}}{\partial t^{2}}
\end{gathered}
$$

By putting above equation in equation (27),

$$
\frac{1}{c^{2}} \frac{\partial^{2} \mathbf{A}}{\partial t^{2}}-\nabla^{2} \mathbf{A}=\mu_{0} \mathbf{J}+\frac{1}{c^{2}} \frac{\partial \mathbf{E}}{\partial t}+\frac{1}{c^{2}} \frac{\partial^{2} \mathbf{A}}{\partial t^{2}}
$$

By using Maxwell's equation (4), in above equation,

$$
\nabla \times \nabla \times \mathbf{A}-\nabla(\nabla \cdot \mathbf{A})=\nabla \times \mathbf{B}
$$

By using equation (16) in above equation,

$$
\nabla \times \nabla \times \mathbf{A}-\nabla(\nabla \cdot \mathbf{A})=\nabla \times \nabla \times \mathbf{A}
$$

$$
-\nabla(\nabla \cdot \mathbf{A})=0
$$

$$
\nabla \cdot \mathbf{A}=0
$$

It is well known that the above equation is of Coulomb gauge. [1, p. 240-242] 


\section{Conclusion}

It is shown that if we formulate equations of scalar and vector potential from generalized wave equations of the electric and magnetic field, we can obtain generalized potential equations. Equations (27) and (28) are generalized potential equations in that it represents a generalization of all gauge theories that we can formulate with potential equations. By choosing different values of "f," we can obtain different gauge theories, as shown in section IV. Thus the origin of gauge theories lies in the generalized potential equations (27) and (28). This method of formulating electromagnetic field theory looks lengthy. However, it provides a clear understanding of the origin of gauge theories and gauge invariance in the teaching of electromagnetic field theory.

As an essential application of generalized potential equations, we can solve equations (27) and (28) for given complicated distribution of charge and current distribution. We can choose the value of "f" such that equations become the easiest to solve. Then by using equations (16) and (19), we can obtain electromagnetic field. So that generalized potential equations (27) and (28) can become very useful in solving complicated electromagnetic problems.

\section{ACKNOWLEDGMENT}

This work has been done independently. The author thanks BoseX, SVNIT research group members for fruitful discussions.

\section{REFERENCES}

[1] J. D. Jackson, Classical electrodynamics, 3rd ed. New York, NY: Wiley, 1999. [Online]. Available: http://cdsweb.cern.ch/record/490457

[2] R. Heras, "The helmholtz theorem and retarded fields," European Journal of Physics, vol. 37, p. 065204, 2016.

[3] R. S. Elliott, "Electromagnetic theory: A simplified representation," IEEE Transactions on Education, vol. 24, no. 4, pp. 294-296, 1981.

[4] B. M. Notaroš, "Geometrical approach to vector analysis in electromagnetics education," IEEE Transactions on Education, vol. 56, no. 3, pp. 336-345, 2013.

[5] C. J. Carpenter, "Teaching electromagnetism in terms of the potentials instead of the 'maxwell' equations," IEEE Transactions on Education, vol. 36, no. 2, pp. 223-226, 1993.

[6] K. F. Warnick, R. H. Selfridge, and D. V. Arnold, "Teaching electromagnetic field theory using differential forms," IEEE Transactions on Education, vol. 40, no. 1, pp. 53-68, 1997.

[7] D. J. Griffiths, Introduction to electrodynamics; 4th ed. Boston, MA: Pearson, 2013, re-published by Cambridge University Press in 2017. [Online]. Available: https://cds.cern.ch/record/1492149]

[8] J. Jackson and L. B. Okun, "Historical roots of gauge invariance," 2001

[9] N. N. B. (Jr.) and A. K. Prykarpatsky, "Modern classical electrodynamics and electromagnetic radiation - vacuum field theory aspects," in Electromagnetic Waves, V. Zhurbenko, Ed. Rijeka: IntechOpen, 2011, ch. 2. [Online]. Available: https://doi.org/10.5772/intechopen.84011

[10] B. Thidé, Electromagnetic Field Theory, 032011.

[11] O. L. Brill and B. Goodman, "Causality in the Coulomb Gauge," American Journal of Physics, vol. 35, no. 9, pp. 832-837, Sep. 1967.

[12] W. Engelhardt, "Gauge invariance in classical electrodynamics," Annales de la Fondation Louis de Broglie, vol. 30, 122005.

[13] D. Petrascheck and R. Folk, "Helmholtz decomposition theorem and blumenthal's extension by regularization," Condensed Matter Physics, vol. 20, p. 13002, 2017.

[14] Y. F. Gui and W. B. Dou, "A rigorous and completed statement on helmholtz theorem," Progress in Electromagnetics Research-pier, vol. 69 , pp. 287-304, 2007

[15] B. Boisseau and C. Barrabès, "Gauge theory in hamiltonian classical mechanics : The electromagnetic and gravitational fields," Journal of Mathematical Physics, vol. 20, no. 10, pp. 2058-2062, 1979. [Online]. Available: https://doi.org/10.1063/1.523972
[16] K.-H. Yang, "The physics of gauge transformations," American Journal of Physics, vol. 73, no. 8, pp. 742-751, 2005. [Online]. Available: https://doi.org/10.1119/1.1938949 